Western University Scholarship@Western

Centre for Decision Sciences and Econometrics

Technical Reports

Economics Working Papers Archive

1985

\title{
On Robustness of Tests of Linear Restrictions in Regression Models with Elliptical Error Distributions
}

Victoria Zinde-Walsh

Aman Ullah

Follow this and additional works at: https://ir.lib.uwo.ca/economicscdse_tr

Part of the Economics Commons

Citation of this paper:

Zinde-Walsh, Victoria, Aman Ullah. "On Robustness of Tests of Linear Restrictions in Regression Models with Elliptical Error Distributions." Centre for Decision Sciences and Econometrics Technical Reports, 8. London, ON: Department of Economics, University of Western Ontario (1985). 


\title{
THE CENTRE FOR DECISION SCIENCES AND ECONOMETRICS
}

On Robustness of Tests of Linear Restrictions in Regression Models with Elliptical Error Distributions

\author{
Victoria Zinde-Walsh \\ Aman Ullah
}

\section{TECHNICAL REPORT NO.8 NOVEMBER 1985}

3

\section{Centre For Decision Sciences And Econometrics}

\section{Social Science Centre}

The University of Western Ontario London, Ontario N6A 5C2

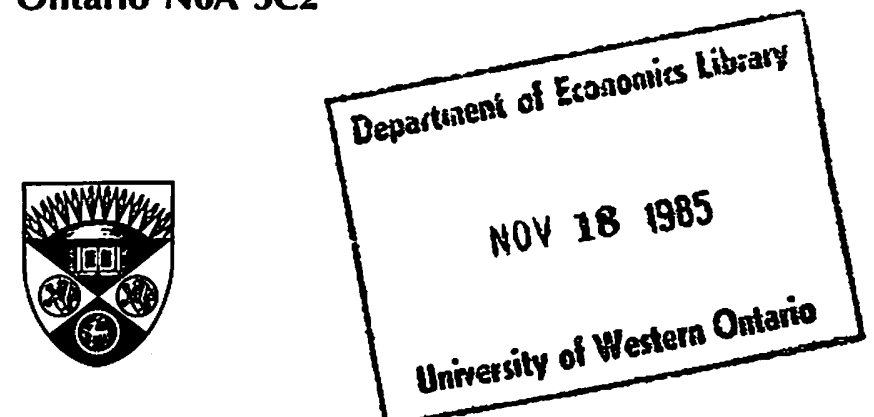


ON ROBUSTNESS OF TESTS OF LINEAR RESTRICTIONS IN

REGRESSION MODELS WITH FILIIPTICAL ERROR DISTRIBUTIONS

by

Victoria Zinde-Walsh and Aman Ullah*

University of Western Ontario

* The authors gratefully acknowledge research support from SSHRC and NSERC, respectively. They are also thankful to $B$. K. Sinha and the members of the U.W.O. Econometrics Workshop for useful comments and discussions on the subject matter of this paper.

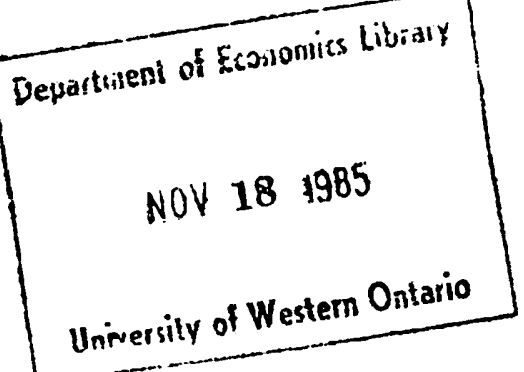


ON ROBUSTNESS OF TESTS OF I.TNHAR RESTRICTIONS IN REGRESSION MODELS

WITH ELLIPTICAL ERROR DISTRIBUTIONS

by

Victoria Zinde.Walsh and Aman Ullah

\section{Introduction}

Testing a set of 1 inear restrictions in a regression model is usually performed with the help of the F-statistic, or the statistic based on the likelihood ratio (LR). More recently two other procedures, the Lagrangian Multiplier or Rao Score (RS) test due to Rao (1947) and Silvey (1959), and the Wald (W) test (1943), have become popular with econometricians; see, for example, Breusch and Pagan (1980) and Evans and Savin (1982).

A statistic can be called numercially robust over a class of error distributions if its values are independent of the specific error distribution from that class. If the statistic is such that no matter which error distribution from the class of distributions is considered, the test criterion remains unchanged then the statistic is inferentially robust over that class.

If the statistics F, LR, RS and $W$ are constructed based on the assumption of the spherical normal error distribution (normal error with the covariance matrix $\sigma^{2} I$ ), then $F$ and LR are numerically robust against the class of all monotonically decreasing continuous spherical distributions, but RS and $W$ are not. However, all these statistics are inferentially robust over this class, thus the test conclusions reached under the assumption of 
normality will not be overturned if the error distribution is spherical. These results are derived by U1lah and Zinde-Walsh (1984), (1985).

In this paper we consider the issues of numerical and inferential robustness of F, LR, RS and $W$ tests, based on the assumption of spherical normality, against the general class of elliptical error distributions (errors with the nonscalar covariance matrix $\Sigma$ ). We provide the necessary and sufficient conditions of numerical robustness for the class of covariance matrices of ten used in econometrics, for example, autoregressive (AR), moving average (MA) and heteroscedasticity. Our investigation shows that for these covariance matrices the numerical robustness of test statistics under consideration is rare. Our results are more general than those given in Ghosh and Sinha (1980) and Sinha and Mukhopadhyaya (1981) who consider only intra-class covariance structure. Also, while Khatri (1981) gave conditions for numerical robustness in terms of pairs of data and covariance matrices, robustness over classes of covariance matrices considered here has not been examined in his paper.

Our investigation also showed the 1 imitations of exact inferential robustness. We therefore looked into the robustness of tests by developing bounds for critical values which will ensure that the conclusions based on the usual tests are not affected against a particular class of distributions. Bounds for critical values of test statistics for $t$ and $F$-tests for first-order AR, MA and ARMA processes have been tabulated (for normal errors) by Vinod (1976), Vinod and Ullah (1981) and Kiviet (1980). Their calculations involve the knowledge of all the eigenvalues of the matrices which characterize these processes and are quite complex. The situation becomes 
more complicated for higher order ARMA processes. Our method of fers bounds which are cruder for the specific processes considered by Vinod and Ullah and Kiviet, but they have the advantage of calculational simplicity and generality, i.e., they provide critical values that guarantee robustness of the test conclusions, for any $\Sigma$ matrix, over wide classes of error distributions, and would utilize only the highest and lowest eigenvalues of the covariance matrix.

The plan of the paper is as follows. Section 2 develops the notation and definitions. Section 3 deals with the problem of numerical robustness and some applications. In Section 4 we examine the question of robustness of test conclusions and provide our bounds on the critical values of statistics. Finally, the proofs of the lemmas and theorems are presented in section 5 .

\section{Definitions and Notation}

We consider the general linear regression model

$$
\text { (2.1) } \quad y=x \beta+u
$$

where $y$ is an $n \times 1$ vector of observations, $x$ is an $n \times p$ known matrix of rank $p<n, \beta$ is a $p \times 1$ unknown parameter vector and $u$ is an $n \times 1$ disturbance vector such that

$$
\phi(u)=\sigma^{-n}|\Sigma|^{-1 / 2} f\left(\frac{u^{\prime} \Sigma^{-1} u}{\sigma^{2}}\right)
$$

with a monotonically decreasing $f$, and positive definite $\Sigma$. If $\Sigma=I$, the distribution $(2.2)$ reduces to a spherically symmetric distribution.

Our problem is to test a set of $r$ linear restrictions $H_{0}: R \beta=0$ against $H_{1}: R \beta: \neq 0$, where $R$ is an $r \times p$ known matrix. Under this hypothesis, following 
Ghosh and Sinha $(1980$, p. 338$)$, we can rewrite the model as

(2.3) $y=x_{0} \beta_{0}+u$,

where $x_{0}$ is an $n \times m$ known matrix of rank $m<p$.

We denote by F, LR, RS, and $W$ the values of the statistics calculated

according to the usual formulae under the assumption of multivariate normality

of $u$. F can be written as

$(2.4)$

$$
F=\left[\frac{(y-X \hat{\beta})^{\prime}(y-X \hat{\beta})}{(y-\hat{\alpha} \beta)^{\prime}(y-\hat{\alpha} \beta)}-1\right] \frac{q}{r}, \quad q=n-p
$$

where $\hat{\beta}_{0}$ and $\hat{\beta}$ are the respective least squares estimators of $\beta_{0}$ and $\beta$, and LR, RS and $W$ can be expressed through $F$, respectively, as

$$
L R=n \log \left(1+\frac{r}{q} F\right), \quad R S=n \frac{r}{q} F /\left(1+\frac{r}{q} F\right), \quad W=n \frac{r}{q} F .
$$

We introduce the following projection matrices:

$$
P=X\left(X^{\prime} X\right)^{-1} X^{\prime} ; A=I-P ; P_{0}=X_{0}\left(X_{0}^{1} X_{0}\right)^{-1} X_{0}^{1} ; A_{0}=I-P_{0} .
$$

where $I$ is the identity matrix. The following properties can be easily verif ied :

(2.7) rank $P>\operatorname{rank} \mathrm{P}_{0}$; $\operatorname{rank} \mathrm{A}<\operatorname{rank} \mathrm{A}_{0}$; $\mathrm{PP}_{0}=\mathrm{P}_{0}$;

$$
A A_{0}=A ; P X=X ; P X_{0}=X_{0}
$$

Using (2.6) and noting that $\left(1+\frac{r}{q} F\right)=\frac{u^{\prime} A}{u^{\prime} A u}$ we can rewrite (2.4)

and $(2.5)$ as

$$
F=\frac{u^{\prime}(A-A) u}{u^{\prime} A u} \frac{q}{r} ; \quad L R=n \log \frac{u^{\prime} A u}{u^{\prime} A u}
$$

$$
R S=n \frac{u^{\prime}(A-A) u}{u_{0}^{\prime} A u} ; \quad W=n \frac{u_{0}^{\prime}(A-A) u}{u^{\prime} A u}
$$


If $u$ is in fact distributed as spherical normal, all the statistics have the known distributions. If the error distribution is spherical, i.e. is given by (2.2) with $\Sigma=I$, we denote the values of the appropriate corresponding statistics by $F_{\phi}, L_{\phi}, R_{\phi}$ and $W_{\phi}$.

If the distribution of $u$ is elliptically normal, we denote the statistics by $F_{\Sigma}, L R_{\Sigma}, R_{\Sigma}$ and $W_{\Sigma}$.

It is known that

$$
F_{\Sigma}=\left[\frac{\left(y-X \hat{\beta}_{0 \Sigma}\right) \cdot \Sigma^{-1}\left(y-X_{0} \hat{\beta}_{o \Sigma}\right)}{\left(y-X \hat{\beta}_{\Sigma}\right) \cdot \Sigma^{-1}\left(y-x \hat{\beta}_{\Sigma}\right)}-1\right] \frac{q}{r}
$$

is the familiar F-statistic for testing $H_{0}$, with

$$
\hat{\beta}_{0 \Sigma}=\left(X_{0}^{1} \Sigma_{0}^{-1} X\right)_{0}^{-1} X \Sigma_{0}^{1} \sum_{i}^{-1} \hat{\beta}_{\Sigma}=\left(X \cdot \varepsilon^{-1} X\right)^{-1} X \cdot \Sigma^{-1} y \text {. }
$$

Further, as in (2.5) we have

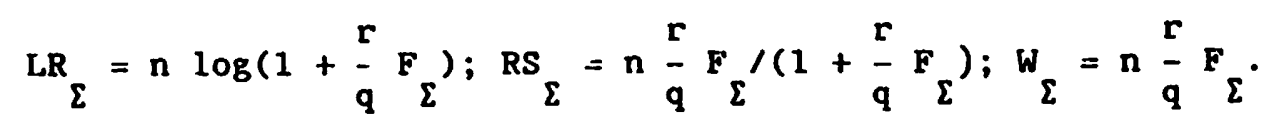

For a general elliptical distribution in (2.2) denote the appropriate statistics by $F_{\phi, \Sigma^{\prime}}, L_{\phi, \Sigma^{\prime}}, R_{\phi, \Sigma^{\prime}}, W_{\phi, \Sigma} \cdot \operatorname{Ullah}$ and Zinde-Walsh (1984, 1985) have analyzed the numerical robustness of LR, RS and $w$ tests against spherically symmetric distributions. In particular, they have shown that

$$
\mathrm{LR}=\mathrm{LR}{ }_{\phi} ; \mathrm{RS}=\Psi_{\phi}^{-1} \mathrm{RS}_{\phi} ; W=P_{\phi}^{-1} W_{\phi} \text {, }
$$

where $\Psi_{\phi}$ and $P_{\phi}$ are constants which depend on the spherical distribution $\phi(u)$. Thus LR is numerically robust but RS and $W$ are not.

The elliptical distribution (2.2) can be transformed into a spherical by the substitution $u=\varepsilon^{2 / 2} v$. Thus for this case, from (2.12) we easily obtain (2.13)

$$
\mathrm{LR}_{\Sigma}=\mathrm{LR}_{\phi, \Sigma} ; \mathrm{RS}_{\Sigma}=\Psi_{\phi}^{-1} \mathrm{RS}_{\phi, \Sigma} ; W_{\Sigma}=\rho_{\phi}^{-1} W_{\phi, \Sigma} \cdot
$$

Here $L R_{\Sigma}$ is numerically robust against non-normality, but $R S_{\Sigma}$ and $W_{\Sigma}$ are not. 


\section{Main Resuits on Numerical Robustness}

It was mentioned in Section 2 that U1lah and Zinde-Walsh (1984, 1985) analyzed the numerical robustness of $F, L R, R S$ and $W$ against spherically symmetric distributions and of $F_{\Sigma}, \operatorname{LR}_{\Sigma}, R_{\Sigma}$ and $W_{\Sigma}$ against elliptically symmetric distributions. Here we look into robustness of F, LR, RS and W (under spherical normal) against elliptical normal distributions by comparing the values of these statistics with the values $F_{\Sigma}, L R_{\Sigma}, R_{\Sigma}$ and $W_{\Sigma}$ as in $(2.9)-(2.11)$.

Conclusions about robustness against general elliptical distributions will follow in view of the relationships (2.13). We also note that we derive the results for parametric classes of $\Sigma$ matrices often used in economic literature.

For deriving the conditions under which $F_{\varepsilon}$ (or $L R_{\Sigma}$ ) is numerically robust over some class of $\Sigma$ given the data matrix $X$, we consider

$$
\text { (3.1) } \ell_{\Sigma}=1+\frac{r}{q} F_{\Sigma}
$$

and examine the conditions under which $\ell_{\Sigma}=\ell_{0}$ for $\ell_{0}=1+\frac{r}{q}$.

Consider a class $\Omega_{p}$ of matrices $\Sigma$ with $\Sigma^{-1}=\left(I-H_{\rho}\right)$ where $H_{p}$ is some symmetric matrix over some parameter space $B \in R^{k}, p=\left(p_{1}, \ldots, \rho_{k}\right) \in B$ with $H_{0}=0$ for $p_{1}=\ldots=p_{k}=0$ and $\left|y^{\prime} H_{\rho} y\right|<y^{\prime} y$ for all possible $y, \rho \in B$. We now state the following lemmas which are used in the proof of Theorem 1. 
Lemma 1

Over all $\Sigma \in \Omega_{\rho}, l_{\Sigma}$ can be represented as

(3.2) $\ell_{\Sigma}=\frac{y^{\prime} A O_{0}+y^{\prime} A T_{\rho}^{O} A}{y^{\prime} A Y+y^{\prime} A T A Y}$

where

$$
T_{\rho}=\Sigma_{i}^{\infty}{ }_{i}+\ldots \Sigma_{1}+i_{m+1}=i^{\left.(-1)^{m+1} H_{\rho}^{i}{ }^{1}{ }^{i}{ }^{2} \ldots A^{A+1}\right]}
$$

and $T_{\rho}$ has a similar representation with $A_{o}$ replacing $A$ in ( 3.3$)$, with $A$ and A defined by $(2.6)$.

\section{Lemma 2}

Suppose that for some symetrix matrix $T$

$$
\left(y^{\prime} A_{0} T_{0} y\right) \cdot\left(y^{\prime} A y\right)=\left(y^{\prime} A T A y\right)\left(y^{\prime} A_{0} y\right) .
$$

Then $A_{0} T A=\theta A_{0} ; T A=\theta A$, where $\theta$ is some constant.

\section{Theorem 1}

Suppose that $H_{p}$ is a polynomial or a convergent series in the parameters $\rho_{1}, \ldots, \rho_{k}$ with symmetric matrices as coefficients. Then if $T\left(r_{1}, \ldots, r_{k}\right)$ is

the coefficient of $\rho^{r}, \ldots, \rho^{k}$ in $\sum_{r=1}^{m_{p} H_{\rho}^{r}, m=\Sigma r}$ and if $\ell_{\Sigma}=\ell$ o

\section{it follows that}

$$
\text { (3.5) } \quad A_{0} T\left(r_{1}, \ldots, r_{k}\right) A_{0}=\theta\left(r_{1}, \ldots, r_{k}\right) A_{0}
$$

for some constant $\theta\left(r_{1}, \ldots, r_{k}\right)$.

For proofs of the lemmas and Theorem 1 see Section 5 . 
Remark 1 Suppose that $A_{0} H_{\rho}^{k} A_{0}=\theta_{k, \rho} A_{0}, k=1,2, \ldots$, where $\theta_{k, \rho}$ is a scalar function of $\rho_{1}, \ldots, \rho_{k}$. Then $\ell_{\Sigma} \equiv \ell_{0}$.

To prove the above statement one only needs to note that

$$
A_{\rho}^{A} A=A A_{0} H_{\rho}^{k} A_{0} A=A \theta_{k, \rho} A_{0}^{A}=\theta_{k, \rho} A
$$

and to substitute into $(3.3),(3.2)$.

Theorem 1 and Remark 1 give the necessary and sufficient conditions for the constancy of $\ell_{\Sigma}$ and therefore for the numerical robustness of $F, R S, L R$ and $W$ statistics against elliptically normal errors that can be described by a variance-covariance matrix $\Sigma \in \Omega_{\rho}, \Omega_{\rho}=\left\{\Sigma \mid \Sigma^{-1}=I-H_{\rho}\right.$ with $H_{P}$ being a polynomial or convergent series\}.

The stringency of these conditions makes numerical robustness an exception rather than the rule. No process with non-trivial $H_{\rho}$ gives rise to robust statistics for all possible $X$ and $X_{0}$, therefore numerical robustness has applications mainly for experimental design. Also, of course, one can always check if the observation matrix $X$ just happened to lead to statistics numerically robust against a particular process in the errors, but if so it would be strictly a matter of luck. We show that our results generalize those on experimental design with intraclass covariance structure by Ghosh and Sinha (1980) and examine the possibilities for numerical robustness over heteroscedastic and ARMA processes.

\subsection{Implications for Intraclass Covariance Structures}

The result of Ghosh and Sinha (1980, Theorem 3.1) follows as a special case of our theorem. Indeed, they considered $\Sigma=(1-p) I+\rho 1_{n} \times 1_{n}^{\prime}$, $-\frac{1}{n-1}<\rho<1$, where $1_{n}$ is a column vector of ones, and hence 
$1_{n} \times 1_{n}^{\prime}=n Q$, where $Q$ is a projector of rank 1 onto the subspace spanned by $1_{n}$. Here $\Sigma^{-1}=\frac{1}{1-\rho}\left[I-\frac{\rho n}{(1+(n-1) \rho)} Q\right]$. Direct application of Theorem 1

to $\Sigma^{-1}$ implies that $A_{0} Q A_{0}=\theta A_{0}$. Since rank $A_{0}>$ rank $A \geq 1$, it follows that $\theta=0, A_{0} 1 \times 1 A_{0}=0, A_{0} 1=0$, therefore $1_{n}$ is the eigenvector of both $P_{0}$ and $P$ as stated in the result of Ghosh and sinha. It is just as easy to verify that Theorem 3.2 of the same paper follows from our results.

\subsection{Implications for Heteroscedastic Errors}

Theorem 1 also provides a characterization of the class of heteroscedastic $\Sigma$ for a given $A$ and restriction $R$ over which $\ell_{\Sigma}=\ell_{0}$ : it is required that $\Sigma=\gamma_{0} I+\Lambda$, where the diagonal matrix $\Lambda$ is such that $\Lambda_{0}=0$, this implies that the A matrix has a block-diagonal structure with a block of zeros.

\subsection{Implications of Theorem 1 for Autoregressive (AR) Error Structures}

The matrix $\Sigma^{-1}$ is known for autoregressive processes of order $k, A R(k)$.

If we set all but one of the parameters of $\operatorname{AR}(k)-\rho_{2}, \rho_{2}, \ldots, \rho_{k}$ equal to zero: $\rho_{k} \neq 0, \rho_{i}=0$ for $i \neq k$, then $\Sigma^{-1}$ reduces to the matrix $I+\rho C_{2 k}+\rho^{2} C_{2 k}$. Here $C_{1 k}$ is the matrix with elements $\left(C_{2 k}\right)_{i j}$ equal to -1 if $|i-j|=k$ and 0 otherwise, and $c_{2 k}$ is a diagonal matrix with elements ( $\left.C_{2 k}\right)_{i j}$ equal to -1 if $k<i=j<n-k$ and 0 otherwise. We shall denote this process $\operatorname{AR}(k, 0)$. A necessary condition for constancy of $\ell_{\varepsilon}$ for $\operatorname{AR}(k, 0)$ is that $A_{0} C_{12} A_{0}=\theta A_{0}$, where $A_{0}$ is a projector of rank no less than 2. This implies that $C_{1 k}$ should have at least two identical eigenvalues, which is true only if $k \geq \frac{n}{2}+1$. If $k \geq \frac{n}{2}+1$ then $c_{2 k}$ has a kernel of dimension $n-2(n-k)=2 k-n \geq 2$. In this case $\ell_{\Sigma}$ is constant for all $A_{0}$ that 
project into the intersection of the kernel of the matrix $C_{2 k}$ and of either the image or the kernel of $C_{2 k}$. Then, of course, $\theta=0$ and $A_{0} C_{2 k} A_{0}=Y A_{0}$ with $Y=0$ or 1 . It is not hard to check that for these $k$ and $A_{0}$ this suffices for constancy of $\ell_{\Sigma}$.

\subsection{Implications for the Moving Average Brror Structure}

The class of MA $(k, 0)$ error structures where all but the parameters of order $\ell$ are zeros is represented by $\varepsilon=\sigma^{2}\left[\left(1+w^{2}\right) I+w C_{1 k}\right]$, where $C_{1 k}$ is the same as for the $A R(k)$ structure. We denote $\sigma^{2}\left(1+w^{2}\right)$ by $Y$ and $w /\left(1+w^{2}\right)$ by $\rho$. Theorem 1 can be directly applied to this class of $\Sigma$ after noting that $\Sigma^{-1}=\left(1+\gamma^{2}\right)\left(I+\rho c_{1 k}+\rho^{2} c_{1 k}^{2}+\ldots\right)$. For this $\Sigma, \ell_{\Sigma}$ is robust only if $A_{0} C_{2 k} A_{0}=\theta A_{0}$. It can be easily seen that for the particular $C_{2 k}$ in the $M A$ error structure $|w| \leq 2$ always. Therefore the theorem applies to all $\rho$ such that $|\rho|<1 / 2$. Since $\rho=w /\left(1+w^{2}\right)$, this condition on $\rho$ is satisfied for any $w$. So the condition $A_{0} C_{2 k} A_{0}=\theta A_{0}$ is necessary and sufficient for robustness over all possible MA error structures.

Here again if $k \geq \frac{n}{2}+1$, matrices $A_{0}$ which result in robust test statistics, exist. Such an $A_{0}$ would project onto the kernel of $\mathrm{C}_{2 \mathrm{k}}$.

Thus we conclude that there are some data structures that produce statistics that are robust over $A R$ and MA error processes of sufficiently high orders (which do not include lower order components). We also notice that the higher the order of the error process the larger the class of data matrices that give robust statistics. This is hardly surprising since in the limiting case processes of order higher than the dimensions of the data will not affect the statistics at all.

We also note that in general the larger the number of equal eigenvalues, including zeros, of $H$ (or the larger the dimension of any projector in the canonical representation of the symmetric matrix $H$ ) the more possibilities for numerically robust statistics. 
Note that if $\ell_{\Sigma}=\ell_{0}$, then $F=F_{\phi, \Sigma}$ and $L R=L_{\phi, \Sigma}$, but unless the distribution is elliptical normal $R S \neq R_{\phi, \Sigma}$ and $W \neq W_{\phi, \Sigma}$.

\section{Inferential Robustness and Bounds on Critical Values}

If two test statistics are such that one is a monotonic function of the other, then any probabilistic statement about one implies a similar statement about the other. Thus if one falls beyond a critical value for some level of the test, so does the other. Therefore, as was stated in U1.1ah and Zinde-Walsh (1985) (and can be seen immediately from (2.12)) RS and $W$ are inferentially robust over the class of all spherical monotonic error distributions .

Here we examine the inferential robustness of the test statistics F, LR, RS and $W$, calculated under the assumption of spherical normality, for general elliptic distributions. To emphasize this we denote the statistics by $F(\Sigma)$, $L R(\Sigma), \operatorname{RS}(\Sigma)$ and $W(\Sigma)$. Since the test statistics are inferentially robust against spherical distributions it will not make any difference to our conclusions whether the statistics bear the subscript $\phi$ or not.

$$
\text { Consider the variate } S(\Sigma)=F(\Sigma) \frac{r}{q} \text {, where }
$$

(4.1) $\quad s(\Sigma)=\frac{U^{\prime} A U}{u^{\prime} A u}$

with $A_{1}=A_{0}-A, A_{2}=A$ as defined in (2.6). The critical values for $S(\Sigma)$ depend on the matrix $\Sigma$. Indeed, consider the transformation $u=\Sigma^{1 / 2} v$, then

(4.2) $\quad S(\Sigma)=\frac{v \cdot \Sigma^{1 / 2} A \sum^{2 / 2} v}{v \cdot \sum_{A}^{1 / 2} \sum_{2}^{1 / 2} v}$

where $v$ is spherically symmetric. Denote $S(I)$ by $S$. 
We observe that as long as $s(\Sigma)$ is inferentially robust over a class $\Omega$ of $\Sigma$ matrices all the statistics $F(\Sigma), L R(\Sigma), R S(\Sigma)$ and $W(\Sigma)$ are inferentially robust over $\Omega$ as well. We assume that $I \in \Omega$.

Denote by $O(n)$ the group of orthogonal $n \times n$ matrices in the Euclidean space $R^{n}$. For any $T \in O(n)$ the distribution of $S$ and of $S(\Sigma)$ in (4.2) is invariant with respect to the transformation $T: R^{n} \rightarrow R^{n}$.

\section{Lerma 3:}

For a positive definite matrix $\varepsilon^{2 / 2}$ and any two mutually orthogonal idempotent matrices $A_{1}, A_{2}$ there exists $T \in O(n)$ such that $\Lambda_{i}=T^{\prime} \Sigma^{2 / 2} A_{i} \Sigma^{1 / 2} T$ is a diagonal matrix for $i=1,2$.

Proof: See Section 5.

This lemma allows us to rewrite $S(\Sigma)$ by substituting $w=$ Tv as

$$
S(\Sigma)=\frac{w^{\prime} w^{\prime}}{w^{\prime} \Lambda_{2}}
$$

where we can write

$$
\Lambda_{1}=\operatorname{diag}\left(\mu_{2}, \ldots, \mu_{k}, 0, \ldots 0\right), \quad \mu_{1} \leq \cdots \leq \mu_{k}, \quad k=p-m
$$

$$
\Lambda_{2}=\operatorname{diag}\left(0, \ldots 0, \mu_{p+1}, \ldots, \mu_{n}\right) \quad \mu_{p+1} \leq \cdots \leq \mu_{n}
$$

where $\operatorname{diag}(. .$.$) denotes a diagonal matrix with given diagonal elements.$

A similar transformation for $s$ yields

$$
\text { (4.5) } \quad S=\frac{w^{\prime} Q^{w}}{w^{\prime} Q_{2} w}
$$

with $Q_{1}=\operatorname{diag}(1, \ldots 1,0, \ldots 0)$, where the first $k$ elements equal 1 , and $Q_{2}=$ $\operatorname{diag}(0, \ldots, 0,1, \ldots 1)$ where the last $n-p$ elements equal 1 . Note that the transformation of $S$ may be performed with a matrix from $O(n)$ different from 
$T$, but the distributions of $S(\Sigma)$ and $S$ are not affected by an orthogonal transformation of the spherical variable.

clearly the following inequality holds

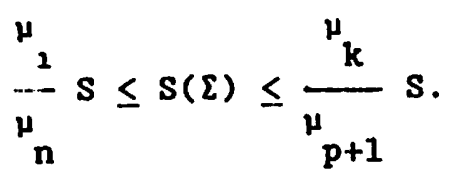

It follows from (4.3) that all the values for $s(\Sigma)$ within the bounds given by (4.6) are realized for some $w$. Therefore a sufficient condition for inferential robustness is that $\mu_{2} / \mu_{2}=\mu_{k} / \mu_{p}$.

However, this type of condition is hardly less restrictive than those demanded for numerical robustness.

We thus seek bounds on the critical values of the statistics $F(\Sigma)$, $\operatorname{LR}(\Sigma), \operatorname{RS}(\Sigma), W(\Sigma)$ which will assure the test conclusions over some class $\Omega(3 \mathrm{I})$ as long as the respective values calculated according to (2.4) and (2.5) are outside of these bounds.

Since $A_{1}, A_{2}$ are projectors with eigenvalues equal to 0 or 1 the eigenvalues of $A \Sigma^{2 / 2}$ are bounded by the eigenvalues of $\varepsilon^{2 / 2}$. Denote $\lambda_{\max }$ the highest and $\lambda_{\min }$ the lowest eigenvalue of $\Sigma$, by $\delta_{\Sigma}$ the ratio $\lambda_{\max } / \lambda_{\min }$. clearly

(4.7) $\quad \lambda_{\min } w^{\prime} Q_{i} w \leq w^{\prime} \Lambda_{i} w \leq \lambda_{\max } w^{\prime} Q_{i} w$.

Therefore

(4.8) $\quad \delta_{\Sigma}^{-1} S \leq S(\Sigma) \leq \delta_{\Sigma} S$.

This inequality holds irrespective of $A_{1}, A_{2}$ and the particular $\Sigma$, and only reflects one characteristic of $\varepsilon$-. the ratio of the highest to lowest eigenvalues. The bigger $\delta_{\Sigma}$ is the more $\Sigma$ is distinguished from $I$ for which $\delta=1$. 
For any two statistics $s_{2}$ and $s_{2}$ with $s_{1} s_{2} s_{2}$ everywhere their cumulative distributions $G_{i}(x)=\operatorname{Prob}\left(s_{i}<x\right) i=1,2$ are related:

$$
G_{1}(x) \geq G_{2}(x) \text {, }
$$

and therefore for some level of the test the critical values satisfy:

$$
s_{1}^{c r} \leq s_{2}^{c r} \text {. }
$$

From this observation and (4.8) we obtain the following theorem.

\section{Theorem 2:}

The critical values $F_{c r}(\Sigma)$ are located within the following intervals dependent on $\delta$--the ratio of highest to lowest eigenvalues of $\Sigma$ :

$$
\text { (4.9) } \quad \delta^{-1} F_{c r} \leq F_{c r}(\Sigma) \leq \delta F_{c r} \text {. }
$$

Corollary

$$
\begin{aligned}
& \mathrm{LR}_{\mathrm{cr}}+\mathrm{u} \log \left(\delta^{-1}-\left(1-\delta^{-1}\right) / \ell_{\mathrm{cr}}\right) \leq \mathrm{LR}(\Sigma) \leq \mathrm{LR}_{\mathrm{cr}}+\mathrm{n} \log \left(\delta-(\delta-1) / \ell_{\mathrm{cr}}\right), \\
& \ell_{\mathrm{cr}}=\exp \left(\operatorname{LR}_{\mathrm{cr}} / \mathrm{n}\right)
\end{aligned}
$$

(4.10)

$$
\frac{\delta^{-1} \operatorname{RS}{ }_{c r}^{n}}{n-\left(1-\delta^{-1}\right) R S} \leq R S(\Sigma) \frac{{ }_{c r}^{R S} c r}{n+(\delta-1) R S}, \delta_{c r}^{-1} W_{c r} \leq W_{c r}(\Sigma) \leq \delta W_{c r} .
$$

The inequalities (4.10) are derived easily from (4.8) and (2.5).

\subsection{Discussion of the Results}

The relationship (4.9) has the following immediate interpretations for the F-test. Firstly, if a class $\Omega$ of $\Sigma$ matrices is such that the biggest ratio of the maximum to minimum eigenvalues of $\Sigma \in \Omega$ is limited by some $\delta$, then the test conclusions are the same for any $\Sigma$ as long as either $F / F_{c r}>\delta$ or $F_{c r} / F>\delta$, where $F$ and $F_{c r}$ are, respectively, the value of the test statistic according to $(2.4)$ and the critical value for the hypothesis 
test under the spherical normal. Secondly, if $F>F_{c r}\left(F<F_{c r}\right)$ then the test conclusions are robust over the class $\Omega$ of $\Sigma$ matrices with $\delta$ the ratios of maximum to minimum eigenvalues, such that $\delta(\Sigma)<F / F_{\mathrm{cr}}(\delta(\Sigma)<$ $\left.\mathrm{F}_{\mathrm{cr}} / \mathrm{F}\right)$.

Since the relationship for $W$ in $(4.10)$ is similar to (4.9) for F the same conclusions apply. A simple examination of (4.10) shows that the bounds on the critical values for $R S(\Sigma)$ are inside the interval $\left[\delta^{-1} \mathrm{RS}_{\mathrm{Cr}}\right.$, $\delta R \mathrm{Cr}_{\mathrm{cr}}$, thus the conclusions made above hold for RS as well.

The following example demonstrates how our bounds compare to those obtained by Vinod (1976) and Vinod and Ullah (1981) for the $t$ statistic under an AR(1) process. Suppose that $\rho=.5$. Then the eigenvalues of the variance-covariance matrix are contained between the asymptotic $(T \rightarrow \infty)$ maximum and minimum eigenvalue, $1+p^{2}+2 p=2.25$ and $1+p^{2}-2 p=.25$. Thus the bounds on the critical value of the $t$ statistic can be calculated based on the square root of the ratio $\sqrt{ } 2.25 / .25=3$. The critical values given by vinod and Ullah are tabulated according to the number of restrictions $p$ and sample size $n$. If $n=50, p=5$, for instance, their Table 4.1 gives 1.14 and 3.93 as the lower and upper bounds, respectively, at the $5 \%$ level, whereas our calculation, which involves only dividing and multiplying by 3 of the standard critical value, gives .671 and 6.042 as the lower and upper bounds, respectively.

However, there are three ways in which our results are an improvement. Firstly, they relate to any $\Sigma$ matrices, not just those generated by an AR(1) or MA(1) process. Secondly, they require the calculation of the maximum and minimum eigenvalues of $\Sigma$ only, whereas vinod and Ullah utilized all the eigenvalues in a much more complicated calculation. Thirdly, our bounds are independent of $A$ and $A_{0}$ matrices. 
Note that if the bounds on the positive eigenvalues of AIA and $\left(A_{0}-A^{-} \sum\left(A_{0}-A\right)\right.$ can be established they will provide more accurate intervals for critical values as can be seen from $(4.6)$ and the fact that

$$
\frac{1}{\mu}>\delta_{\Sigma}^{-1} \text { and } \frac{\mu_{k}}{\mu_{p}} \leq \delta_{\Sigma} \text {. }
$$

Recall that under the multivariate normal error distributions Evans and Savin (1982) have derived for $|\mathrm{W} / \mathrm{n}|<1$ the following relationship

$$
\text { (4.11) } W-L R \simeq L R-R S \simeq W^{2} / 2 n
$$

generalizing the known inequality

$$
\text { (4.12) } \quad W \geq L R \geq R S \text {. }
$$

U1lah and Zinde-Walsh (1984) have shown that a more complex relationship exists between the statistics $W_{\phi}, \mathrm{LR}_{\phi}, \mathrm{RS}_{\phi}$ when the distribution is spherical but non-normal. Here, once again, straightforward inequalities relating the bounds on the statistics can be derived.

For any of the statistics F, LR, RS or W, denote by an upper or lower bar the upper or lower bound, correspondingly, given by (4.9) and (4.10). Next we denote

$$
\text { (4.13) } \quad F_{U}=(\bar{F}-\underline{F}) / \bar{F} \text { and } F_{L}=(\bar{F}-\underline{F}) / \underline{F} \text {. }
$$

Similarly, we define $L R_{U}$ and $L R_{L}, R S_{U}$ and $R S_{L}, W_{U}$ and $W_{L}$. These ratios show the length of the interval between the bounds in relation to its upper and lower point, respectively. Thus they measure the "tightness" of the bounds on the critical values of the statistics and the following theorem establishes a ranking of the statistics with respect to this characteristic. 


\section{Theorem 3:}

\section{The following relationships hold}

$$
\text { (4.14) } \quad F_{U}=W_{U} \geq L \cdot R_{U} \geq R S_{U} ; \quad F_{L}=W_{L} \geq L R_{L} \geq R S_{L} \text {. }
$$

\section{Proof:}

\section{See Section 5 .}

This theorem demonstrates the relative robustness of the bounds on critical values for the different statistics. The bounds are the tightest for RS and are the worst when the F test or the $W$ test is used.

\section{Proofs of the Lemmas and Theorems}

\section{Proof of Lemma 1}

From (2.9) and (3.1) we can write

$$
\ell_{\Sigma}=\frac{\left(y-X \hat{\beta}_{0 \Sigma}\right) \cdot \Sigma^{-1}\left(y-X \hat{\beta}_{0}\right)}{\left(y-X \hat{\beta}_{\Sigma}\right) \cdot \Sigma^{-1}\left(y-X \hat{\beta}_{\Sigma}\right)} .
$$

We transform the denominator of (5.1) by substituting

$\hat{\beta}_{\varepsilon}=\left(X \cdot \Sigma^{-1} X\right)^{-1} X^{\prime} \Sigma^{-1} y$ and get

(5.2) $\quad y \cdot \Sigma^{-1}\left[I-X\left(X \cdot \Sigma^{-2} X\right)^{-2} X \Sigma^{-1}\right] y$.

Consider

(5.3) $\quad \Sigma^{-1}-\Sigma^{-1} X\left(X^{\prime} \Sigma^{-1} X\right)^{-1} X \cdot \Sigma^{-1}$

where $\Sigma^{-1}=(I-H)$ with $\left|y^{\prime} H y\right|<y^{\prime} y$.

We can expand part of (5.3) into a geometric series:

$$
\begin{aligned}
X\left[X^{\prime}(I-H) X\right]^{-1} X^{\prime} & =X\left(X^{\prime} X\right)^{-1 / 2}\left[I-\left(X^{\prime} X\right)^{-2 / 2} X^{\prime} H X\left(X^{\prime} X\right)^{-1 / 2}\right]^{-1}\left(X^{\prime} X\right)^{-1 / 2} X^{\prime} \\
& =P+\text { PHP }+ \text { PHPHP }+\ldots+P(H P)^{k}+\ldots
\end{aligned}
$$

where $P$ is defined by (2.6). Substituting into (5.3) we get I - H - P . PHP - PHP - .. $-\mathrm{P}(\mathrm{HP})^{k}-\ldots+\mathrm{HP}+\mathrm{HPHP}+\ldots$

$+(\mathrm{HP})^{k}+\ldots-\mathrm{HPH}-\mathrm{HPHPH}-\ldots-\mathrm{H}(\mathrm{PH})^{k}-\ldots+\mathrm{PH}+\mathrm{PHPH}+\ldots$

$+(\mathrm{PH})^{k}+\ldots=\mathrm{A}-\mathrm{AHA}-\mathrm{AHF} H \mathrm{~A}-\ldots-\mathrm{AH}(\mathrm{PH})^{\mathrm{k}} \mathrm{A}-\ldots$ 
Indeed the last term is obtained in the following way:

$-P(H P)^{k} H P+(H P)^{k} H P-H(P H)^{k}+P(H P)^{k} H=-A H(P H)^{k} A$

(we have substituted $P=I-A$ ). We therefore get that (5.3) can be represented

as

(5.4) A - AHA - AHPHA - .. AH $(\mathrm{PH})^{k} \mathrm{~A}-\ldots$

Further we can replace $P$ by I-A everywhere in (5.4) to get

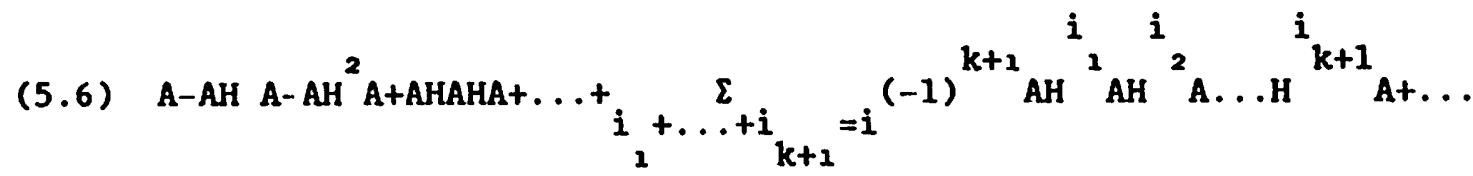

This formula can be easily verified by substitution of $P=I-A$ into (5.4). The numerator of $\ell_{\varepsilon}$ can be transformed in an analogous manner. This concludes the proof of Lemma 1 .

Proof of Lemma 2

For a given vector $y$ we define mutually orthogonal unitary vectors $y_{0}$, $y_{1}$ and $y_{2}$, such that $y_{i}^{\prime} y_{i}=1, i=0,1,2 ; y_{i}^{\prime} y_{j}=0$ for all $0 \leq i<j \leq 2$;

$$
A y=\alpha y_{1} ;\left(A_{0} \cdot A\right) y=B y_{0} ; P_{0} y=Y y_{2} \cdot
$$

By substituting into (3.4) we get

$$
\alpha^{2}\left(\alpha^{2} y_{1}^{\prime} \mathrm{Ty}_{1}+\alpha \beta y_{2}^{\prime} T y_{0}+\alpha \beta y_{0}^{\prime} \mathrm{Ty}_{2}+\beta^{2} y_{0}^{\prime} T y_{0}\right)=\left(\alpha^{2}+\beta^{2}\right)\left(\alpha^{2} y_{1}^{\prime} \mathrm{Ty}_{2}\right)
$$

We equate the coefficients of all the monomials in $\alpha$ and $\beta$ in (5.7)

and get

$$
\begin{aligned}
& \text { (5.8) } \mathrm{y}_{2}^{\prime} \mathrm{Ty}_{2}=\mathrm{y}_{0}^{\prime} \mathrm{Ty}_{0}, \\
& \text { (5.9) } \mathrm{y}_{1}^{\prime} \mathrm{Ty}_{0}+y_{0}^{\prime} \mathrm{Ty}_{1}=0 .
\end{aligned}
$$

Since $T$ is symmetric, (5.9) implies

$$
\mathrm{y}_{1}^{\prime} \mathrm{Ty} y_{0}=y_{0}^{\prime} \mathrm{Ty} y_{1}=0 \text {. }
$$

Conditions (5.8) and (5.10) hold for any $y$. We can denote $y_{1}^{\prime}$ Ty $_{2}$ by $\theta$, where $\theta$ is a constant. For any $y$, 


$$
y_{1}^{1} \mathrm{Ty}_{1}=y^{\prime} \operatorname{ATAy} / \alpha^{2}=\theta
$$

where $\alpha^{2}=y^{\prime} A y$, and we have $y^{\prime} A T A y=\theta y^{\prime} A y$.

similarly, using (5.10) in addition to (5.8), we can show that

$$
y^{\prime} A_{0} T A_{0} y=\theta y^{\prime} A_{0} y \text {. }
$$

\section{Proof of Theorem 1}

Consider the expression (3.2) for $\ell_{\Sigma}$. We can write it as

$$
\begin{aligned}
& 1+y^{\prime} A T^{O} A y^{\prime} / y^{\prime} A y \\
& \text { (5.11) } \ell_{\Sigma}=\ell_{0} \frac{0 \rho \circ}{1+y^{\prime A T} \text { Ay/ } y^{\prime} \text { Ay }}
\end{aligned}
$$

If $l_{\Sigma}=l_{0}$ it follows that

$$
\left(y^{\prime} A_{0} T_{P_{0}^{\circ}}^{O} A_{0} y\right) y^{\prime} A y=\left(y^{\prime} A T{ }_{p}^{A y}\right) y^{\prime} A_{0} y \text {. }
$$

The expressions on each side of (5.1.2) are series in the parameters $p_{1} \ldots$ $\rho_{k}$ of $H_{\rho}$. If the two series of the right and left sides in (5.12) are to be identical all the coefficients of monomials have to coincide. Consider $P_{1}$ and the coefficient of the lowest power of $\rho_{1}$ in $T_{p}$, it is some symmetric matrix $T_{1}$ (it is also the coefficient of $P_{1}$ in $H_{\rho}$ ).

We get for $T_{1}$

$$
\left(y^{\prime} A_{0} T_{2} A_{0} y\right) y^{\prime} A y=\left(y^{\prime} A T_{2} A y\right) y^{\prime} A_{0} y
$$

thus by Lemma 2 ,

$$
A_{0} T_{1} A_{0}=\theta_{1} A \text { (and } A T_{1} A=\theta_{1} A \text { ). }
$$

Similar equalities hold for all coefficients of lowest powers in $T_{p}$. 
Any coefficient of a monomial $\rho_{2}^{{ }_{2}}, \ldots, \rho^{{ }^{k}}$ in $T_{\rho}^{\circ}$ can be

represented as the sum of such a coefficient in $\sum_{r=1}^{r+r}+\ldots+r_{k} r_{H}$

denoted by $T\left(r_{1}, \ldots, r_{k}\right)$, and products of coefficients of lower power monomials with $A_{0}$ in between. We can now use induction to show that

$$
A_{0} T\left(r_{1}, \ldots, r_{k}\right) A_{0}=\theta\left(r_{1}, \ldots, r_{k}\right) A_{0} .
$$

If (5.13) holds for all coefficients of monomials of lower power, we can replace such $A_{0} T A_{0}$ by the appropriate $\theta A_{0}$ and will arrive at (3.4).

This concludes the proof of Theorem 1 .

\section{Proof of Lemma 3:}

Let $T_{i} \in O(n)$ be a matrix that diagonalizes $\Sigma^{1 / 2} A_{i} \Sigma^{1 / 2}: T_{i}^{1} \Sigma^{1 / 2} A_{i} \Sigma^{1 / 2} T_{i}=\Lambda_{i}$, where $\Lambda_{i}$ is diagonal. Denote by $Q_{i}$ the orthogonal projector onto the space of non-zero eigenvectors of $\Sigma^{3 / 2} A_{i} \Sigma^{1 / 2}$.

We show that $Q_{1}$ and $Q_{2}$ are mutually orthogonal, thus each $T_{i}$ can be represented by the same matrix $M_{1}+M_{2}+M_{3}$ with $M_{i}$ mutually orthogonal, the columns of $\mathrm{M}_{2}\left(\mathbb{M}_{2}\right)$ formed by the orthonormal system of non-zero eigenvectors of $\Sigma^{1 / 2} A_{2} \Sigma^{1 / 2}\left(\Sigma^{1 / 2} A_{2} \Sigma^{1 / 2}\right)$. Suppose that for some vector $\xi, \Sigma^{1 / 2} A_{i} \Sigma^{1 / 2} \xi=\lambda \xi$ with $\lambda$ $\neq 0$, then $Q_{i} \xi=\xi$. We have $A_{i} \Sigma^{1 / 2} \xi=\lambda \Sigma^{-1 / 2} \xi=A_{i}^{2} \Sigma^{1 / 2} \xi=\lambda A_{i} \Sigma^{-1 / 2} \xi$, therefore $A_{i} \Sigma^{-1 / 2} \xi=\Sigma^{-1 / 2} \xi$. Clearly then for any $n$ such that $n=Q_{i} n$ we have $A_{i} \Sigma^{-1 / 2} \eta=\Sigma^{-1 / 2} Q_{i} \eta$. For a vector $\eta$ for which $Q_{1} n=Q_{2} \eta=\eta$ one would have $\eta=\Sigma^{1 / 2} A_{2} \Sigma^{-1 / 2} \eta=\Sigma^{1 / 2} A_{1} \Sigma^{-1 / 2} n=\left(\Sigma^{1 / 2} A_{2} \Sigma^{-1 / 2}\right)\left(\Sigma^{1 / 2} A_{1} \Sigma^{-1 / 2}\right) \eta=n=0$ since $A_{2} A_{1}=0$.

Therefore $Q_{2}$ and $Q_{2}$ are orthogonal projectors.

This concludes the proof.

\section{Proof of Theorem 3}

Represent all the bounds as functions of $s=s_{c r}$, by combining (2.11), $(4.9)$ and $(4.10)$. 


$$
\bar{F}=(q / r) \delta S ; \bar{W}=n \delta S ; \overline{L R}=n \log (1+\delta S) ; \overline{R S}=n \delta S /(1+\delta S) ;
$$

$$
\underline{E}=(q / r) \delta^{-1} S ; W=n \delta^{-1} S ; \underline{L R}=n \log \left(1+\delta^{-1} S\right) ; \underline{R S}=n \delta^{-1} S /\left(1+\delta^{-1} S\right) .
$$

Next we derive directly that

$$
\begin{array}{ll}
\text { (5.15) } & F_{U}=W_{U}=1-\delta^{-2} ; F_{L}=W_{L}=\delta^{2}-1 ; \\
\text { (5.16) } & W_{U}-L R_{U}=\ln \left(1+\delta^{-1} S\right) / \ln (1+\delta S)-\delta^{2} \\
\text { (5.17) } & W_{L}-L R_{L}=\delta^{2}-\ln (1+\delta S) / \ln \left(1+\delta^{-1} S\right) ; \\
\text { (5.18) } & L_{U}-R_{U}=\delta^{-2}(1+\delta S) /\left(1+\delta^{-1} S\right)-\ln \left(1+\delta^{-1} S\right) / \ln (1+\delta S) \\
\text { (5.19) } \quad L_{L}-R_{L}=\ln (1+\delta S) / \ln \left(1+\delta^{-1} S\right)-\delta^{2}\left(1+\delta^{-1} S\right) /(1+\delta S) .
\end{array}
$$

It immediately follows from (5.15) that whatever conclusions will be proved to hold here with respect to $W$ will apply to $F$ as well.

Examine (5.16). The expression $\ln \left(1+\delta^{-1} S\right)-\delta^{-2} \ln (1+\delta S)$ is always non-negative since it equals zero for $S=0$ and its derivative with respect to $S$ is $\frac{\delta^{-1}\left(\delta S-\delta^{-1} S\right)}{\left(1+\delta^{-1}\right)(1+\delta S)}$ and is thus positive. This proves that (5.16) is positive for positive S. Similarly, we show that $\delta^{2} \ln \left(1+\delta^{-1} S\right)-\ln (1+\delta S)$ is positive for $s>0$, thus (5.17) is positive. Next, consider the expression

$$
\delta^{-2}(1+\delta S) \ln (1+\delta S)-\left(1+\delta^{-1} S\right) \ln \left(1+\delta^{-1} S\right)
$$

related to $(5.18)$. It is zero for $S=0$, its derivative is equal to $\delta^{-1} \ln \frac{1+\delta S}{1+\delta^{-1} S}$ and positive. Therefore (5.18) is positive. Similarly from $(1+\delta S) \ln (1+\delta S)-\delta^{2}\left(1+\delta^{-1} s\right) \ln \left(1+\delta^{-1} s\right)$ being positive (identical proof) it follows that $(5.19)$ is positive. This concludes the proof of Theorem 3 . 
REFERENCES

Evans, G. B. A. and N. E. Savin: "Conflict Among the Criteria Revisited: The W. LR, and LM Tests," Econometrica 50 (1982), 737-748.

Ghosh, M. and B. K. Sinha: "On the Robustness of Least Squares Procedures in Regression Models," Journal of Multivariate Analysis 10 (1980), 332-342.

Khatri, C. G.: "Study of F--Tests Under Dependent Models," The Indian Journal of Statistics 43 (1981), A, 107-110.

Kiviet, J. F.: "Effects of ARMA Errors on Tests for Regression Coefficients: Comments on Vinod's Article; Improved and Additional Results," Journal of the American Statistical Association 75 (1980).

Rao, C. R.: "Large Sample Tests of Statistical Hypotheses Concerning Several Parameters with Applications to Problems of Estimation," Proceedings of the Cambridge Philosophical Society 44 (1974), 50-57.

Savin, N. E.: "Conflict Among Testing Procedures in a Linear Regression Model With Augoregressive Disturbances," Econometrica 44 (1976), 1303-1315.

silvey, S. D.: "The Lagrange Multiplier Test," Annals of Mathematical Statistics 30 (1959), 389-407.

Sinha, B. K. and B. B. Mukhopodhyay: "A Note on a result of Ghosh and Sinha," Calcutta Statistical Association Bulletin (1981), 169-177.

U1lah, A. and V. Zinde-Walsh: "On the Robustness of LM, LR and W Tests in Regression Models," Econometrica 52 (1984), 1055-1066. : "Estimation and Testing in Regression Models with Spherically Symmetric Errors," Economics Letters 17 (1985), 127-132. 
Vinod, H. D.: "Fffects of ARMA Frrors on the Significance Tests for Regression Coefficients," Journal of the American Statistical Association 71 (1976), 929-933.

Vinod, H. D. and A. Ullah: Recent Advances in Regression Methods. New York: Marce1 Dekker, 1981.

Wald, A.: "Tests of Hypotheses Concerning Several Parameters When the Number of Observations is Large," Transactions of the American Mathematical Society 54 (1943), 426-482. 